\title{
Análisis de las redes sociales en el consumo de alcohol
}

\author{
María del Carmen Pérez-Fuentes ${ }^{1,2}$, María del Mar Molero ${ }^{1}$, \\ José Jesús Gázquez ${ }^{1,3}$, María del Mar Simón ${ }^{1}$, África Martos ${ }^{1}$, \\ Ana Belén Barragán ${ }^{1}$, e Isabel Mercader ${ }^{1}$ \\ ${ }^{1}$ Universidad de Almería (España); ${ }^{2}$ Universidad Politécnica y Artística \\ del Paraguay (Paraguay); ${ }^{3}$ Universidad Autónoma de Chile (Chile)
}

\begin{abstract}
El consumo de alcohol se ha convertido en un problema grave de salud, en la actualidad, entre los jóvenes de 14 y 30 años. El objetivo del presente trabajo fue analizar la comunicación en redes sociales en relación al consumo de alcohol. Durante el periodo de tiempo descrito se recopilaron un total de 2063 comentarios de Twitter. Los resultados muestran las diferentes opiniones que tienen los usuarios, los medios divulgativos, así como la policía o los grupos de protesta sobre la ingesta de alcohol. De modo, que la mayoría de jóvenes apoyan el consumo de alcohol como forma de divertirse, mientras que la policía, los grupos de protesta y los medios de divulgación muestran a la sociedad y concretamente a los jóvenes las consecuencias que tiene el consumo. Como conclusión, cabe destacar como las redes sociales se han convertido en la nueva forma de comunicarse entre los jóvenes, lo que facilita el intercambio de opiniones, ideas e información a nivel mundial. Así, han posibilitado observar como las redes sociales influyen en el consumo y la prevención del alcohol, por tanto, las redes pueden ser utilizadas como herramientas para realizar campañas y programas de prevención del consumo de alcohol en los jóvenes, reduciendo la ingesta del mismo.
\end{abstract}

Palabras clave: Alcohol, consumo, prevención, redes sociales.

Analysis of social networks in alcohol consumption. Alcohol consumption has become a serious health problem, today, among young people between 14 and 30 years old. The objective of this study was to analyze communication in social networks in relation to alcohol consumption. During the period of time described, a total of 2063 Twitter comments were collected. The results show the different opinions that users, the media, as well as the police or protest groups have about alcohol consumption. Thus, the majority of young people support the consumption of alcohol as a way of having fun, while the police, protest groups and the media show society and specifically young people the consequences of consumption. In conclusion, it should be noted how social networks have become the new way of communicating among young people, which facilitates the exchange of opinions, ideas and information worldwide. Thus, they have made it possible to observe how social networks influence alcohol consumption and prevention, therefore, networks can be used as tools to carry out campaigns and programs for the prevention of alcohol consumption in young people, reducing their intake.

Keywords: Alcohol, consumption, prevention, social networks.

Correspondencia: $\mathrm{M}^{\mathrm{a}}$ del Carmen Pérez-Fuentes. Departamento de Psicología. Universidad de Almería. Ctra. Sacramento s/n. C.P.:04120. Almería (España). E-mail: mpf421@ual.es 
En la actualidad, el consumo de alcohol se ha convertido en un problema grave de salud a nivel mundial, relacionado con trastornos mentales, cáncer, enfermedades de origen cardiovascular, y una elevado porcentaje de muertes derivadas del consumo nocivo de alcohol (OMS, 2018). De forma general, cabe destacar que las personas que ingieren alcohol de manera moderada no tiene un impacto directo en la salud, sin embargo, un consumo excesivo de alcohol puede influir en el aumento de posibles enfermedades relacionadas con este incipiente consumo (Piano, 2017; Molina y Nelson, 2018). Estos problemas de salud no vienen derivados exclusivamente de personas adictas, sino también de aquellas personas que abusan del alcohol de manera temporal o eventual (Zadarko-Domaradzka et al., 2018).

El consumo de alcohol es común entre los jóvenes de 14 y 30 años (ESTUDES, 2019; Martínez-Loredo, Fernández-Hermida, de la Torre-Luque, y Fernández-Artamendi, 2018; Parada et al., 2011). Es durante esta etapa donde se muestran elevados niveles de ingesta de alcohol, concretamente en su etapa universitaria. La vida de los jóvenes experimenta un cambio al sentirse más independientes y menos supervisados por sus padres, facilitando así, las relaciones sociales con su entorno cercano y fomentando el consumo de alcohol. A menudo, el consumo de alcohol se convierte en una actividad social, desarrollando en consecuencia su identidad. La elevada ingesta de alcohol de manera frecuente conlleva problemas psicológicos y físicos y es el resultado de la relación entre factores personales, ambientales y sociodemográficos (Hall et al., 2016; Lu et al., 2019; Pérez-Fuentes et al., 2018; Sebena, Orosova, Mikolajczyk, y Van Dijk, 2011).

El alcohol una de las sustancias psicoactivas más propagadas y aceptadas socialmente en todos los grupos de edad, aunque su consumo resulte nocivo para la salud (Rehm et al., 2017). El consumo de alcohol en los jóvenes, se ha asociado con factores sociales, familiares y escolares, entre los que encontramos la actitud liberal de los padres hacia la ingesta de alcohol (Cutrín, Maneiro, Sobral, y Gómez-Fraguela, 2019; Murphy, O'Sullivan, O'Donovan, Hope, y Davoren, 2016), el consumo de alcohol por parte de los padres (Jorge et al., 2017), la presión de grupo y la influencia del grupo de iguales en la conducta e ingesta de alcohol, el nivel socioeconómico de los mismos, así como el escaso interés mostrado por los estudios, el absentismo escolar, y la escasa implicación escolar (Alonso-Fernández, Jiménez-Trujillo, Hernández-Barrera, Palacios-Ceña, y Carrasco-Garrido, 2019; De la Fuente, Rodríguez, y Escalante, 2019; Huertas, López-Moreno, Fernández, Echeverry-Alzate, y Bühler, 2019).

Por otro lado, el consumo de alcohol se relaciona con conductas violentas e impulsivas, así como a conductas de riesgo psicosocial en los que los jóvenes con una baja inteligencia emocional como consecuencia de un bajo rendimiento académico y un bajo apoyo de la familia y de las amistades, son propensos a ingerir alcohol (Fernández-Lasarte, Ramos-Díaze, y Axpe, 2019; Gázquez et al., 2015; Moore et al., 
2017; Pérez-Fuentes et al., 2015). Por todo ello, se hace necesario desarrollar estrategias para la prevención e intervención del consumo (Bowden, Delfabbro, Room, Miller, y Wilson, 2017; López y Rodríguez-Arias, 2010).

El Binge Drinking en los jóvenes suele aumentar aprovechando días festivos (fiestas navideñas, puentes, entre otros), así como los eventos deportivos, vacaciones y diversas ocasiones en las que los jóvenes tienen la posibilidad de ingerir alcohol. Este tipo de ingesta, caracterizada por el consumo de alcohol de manera descontrolada, tienen consecuencias perjudiciales para la salud (Bellis et al., 2015; Maganto, Peris, y Sánchez, 2019).

Con el fin de paliar el consumo de alcohol, el Estado pone en práctica diferentes medidas para regular el uso del alcohol, como el aumento de los impuestos en bebidas alcohólicas (Dumont et al., 2017; Meier et al., 2016; Nelson y McNall, 2016). Además, en España la Ley 5/2018, de 3 de mayo, de prevención del consumo de bebidas alcohólicas en la infancia y la adolescencia, pretende regular la venta y el consumo de alcohol en menores aplicando para ello, sanciones y medidas restrictivas.

Asimismo, el gobierno realiza a diario campañas de consumo responsable con la finalidad de reducir los daños que genera el consumo de alcohol y mejorar en consecuencia la salud pública. Este tipo de campañas se realizan a través de anuncios de prevención en televisión y mediante mensajes en carretera (Kersbergen y Field, 2017; Stautz, Brown, King, Shemilt, y Marteau, 2016).

\section{Consumo de alcohol y redes sociales}

La forma de socializarse entre los jóvenes ha cambiado debido a las nuevas tecnologías, pasando cada vez más tiempo comunicándose en línea, donde las redes sociales tienen un papel importante en la vida cotidiana de los jóvenes (Moreno, D'Angelo, y Whitehill, 2016). Diversos estudios muestran como las redes sociales como Facebook, Twitter o Instagram son utilizadas habitualmente para expresar opiniones acerca del consumo de alcohol (Beullens y Schepers, 2013; Moreno, Christakis, Egan, Brockman, y Becker, 2012).

Diversos estudios muestran como las redes sociales pueden llegar a fomentar el consumo de alcohol, debido a que los usuarios realizan de manera frecuente comentarios positivos sobre la ingesta de alcohol (Boyle, LaBrie, Froidevaux, y Witkovic, 2016; Moreno, Cox, Young, y Haaland, 2015), existiendo una relación evidente entre el uso de las redes sociales y beber alcohol (Hendriks, Van den Putte, Gebhardt, y Moreno, 2018).

La demanda de entradas sobre el consumo de alcohol se ha visto incrementada durante las diferentes festividades y fines de semana, donde los jóvenes publican tweets o entradas de Facebook relacionadas con el alcohol expresando opiniones positivas hacia el consumo del mismo (Cavazos-Rehg, Krauss, Sowles, y Bierut, 2015; West et al., 
2012). No obstante, hay múltiples usuarios de Facebook y Twitter que muestran opiniones negativas acerca del consumo de alcohol y las consecuencias que tiene, aunque en menor proporción respecto a los que se muestran a favor de la ingesta (Litt et al., 2018).

Así mismo, las redes sociales facilitan la intervención en la prevención de consumo de alcohol, ya que proporciona información imprescindible sobre el papel que juega la ingesta de alcohol en los jóvenes y ofrece diferentes medios para identificar los individuos que requieren de una intervención con respecto a su consumo elevado de alcohol (Litt et al., 2018).

\section{Visión actual del trabajo}

A partir de la revisión de la literatura, sobre la presencia de comentarios sobre el consumo de alcohol en redes sociales, se pone de manifiesto la importancia de las redes sociales en la comunicación de los jóvenes, aumentando la producción científica de trabajos relacionados con la temática del presente trabajo. De esta manera, se busca mostrar nuevos datos sobre el efecto que tiene en los jóvenes el acceso a redes sociales en el consumo de alcohol.

Por todo ello, el objetivo del presente trabajo es analizar la comunicación en redes sociales en relación al consumo de alcohol.

Avanzando en la evidencia teórica expuesta anteriormente, se consideran las siguientes hipótesis: 1. Los usuarios de las redes sociales se muestran a favor del consumo de alcohol. 2: Los días festivos aumentan los comentarios acerca del consumo del alcohol.

\section{METODOLOGÍA}

\section{Conjunto de datos}

El presente trabajo utilizó software Netlytic para la recopilación de Tweets. Dicha recopilación se realizó entre el 23 de diciembre de 2019 y el 2 de diciembre de 2019. El programa utilizado extrajo todos aquellos mensajes que contuviesen los descriptores "botellón", "alcohol”, "bebida" y "borracho", limitando el idioma a español e inglés. Durante el periodo de tiempo descrito se recopilaron un total de 2063 comentarios de Twitter.

\section{Análisis de datos}

Para la preparación de datos y el análisis de los comentarios de Twitter, se utilizó ATLAS.ti software (versión 8.4, Scientific Software Development), se categorizaron las entradas en función de la temática tratada sobre el consumo de alcohol, así como los comentarios a favor y en contra de la ingesta de alcohol. Para ello, se excluyeron aquellas entradas que no estaban relacionadas con el consumo de alcohol o 
que no proporcionaban información relevante sobre el alcohol, además, se eliminaron los retweets compartidas. Tras aplicar los diferentes criterios de exclusión se eliminó el $79.84 \%$, quedando con un total de 480 comentarios.

Tabla 1. Recopilación de comentarios en función del medio obtenido

\begin{tabular}{lc}
\hline Medio de información & Número de Comentarios \\
\hline Usuarios & 452 \\
\hline Medio de divulgación & 11 \\
\hline Grupos de protesta & 3 \\
\hline Policía & 14 \\
\hline Total & 480 \\
\hline
\end{tabular}

En la tabla 1, se muestran los comentarios hallados en función de la fuente procedente, entre los que destacamos usuarios (452), policías (14), medios de divulgación (11), seguidos de grupos de protestas en menor proporción.

\section{RESULTADOS}

Tal y como podemos observar en la figura 1, son diversos los Tweet encontrados en los que se expresan diferentes opiniones con respecto al consumo del alcohol. Se puede observar cómo hay un porcentaje más elevado de comentarios y opiniones a favor del consumo de alcohol, encontrando un total de 281 tweet. Por otro lado, se muestran comentarios en los cuales alertan de las consecuencias de la ingesta de alcohol de forma continuada en función del colectivo analizado, en el caso de los usuarios se encuentran 199 tweet en contra del consumo de alcohol, se muestra que en un porcentaje menor son los cuerpos de seguridad los que alertan de las graves consecuencias que puede tener el abuso del consumo de alcohol (14), así como los medios de difusión (11), y grupos de protesta en contra del consumo de alcohol (3).

Figura 1. Tipos de comentarios por parte de los usuarios de Twitter

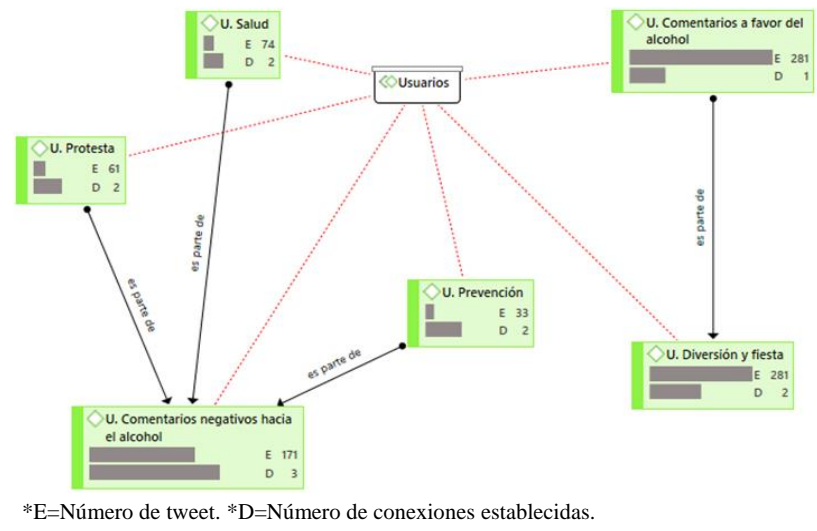


Por otro lado, en la figura 2, se muestran las diferentes opiniones que muestran los usuarios sobre la ingesta de alcohol, encontrando un elevado número de tweets que expresan comentarios positivos sobre el consumo de alcohol, los cuales relacionan el ingerir alcohol con un motivo de fiesta y diversión (281). Por el contrario, se han analizado aquellos tweets que muestran una opinión negativa sobre la ingesta de alcohol, manifestando en las redes los efectos negativos que tiene para la salud (74), mostrando el descontento por los efectos negativos que tiene el botellón en la vía pública como es los residuos que genera, el ruido y en ocasiones violencia (61), y, por último, realizan publicaciones con el objetivo de hacer campañas para prevenir el consumo de alcohol (33).

Figura 2. Tipos de comentarios por parte de los medios de divulgación en Twitter

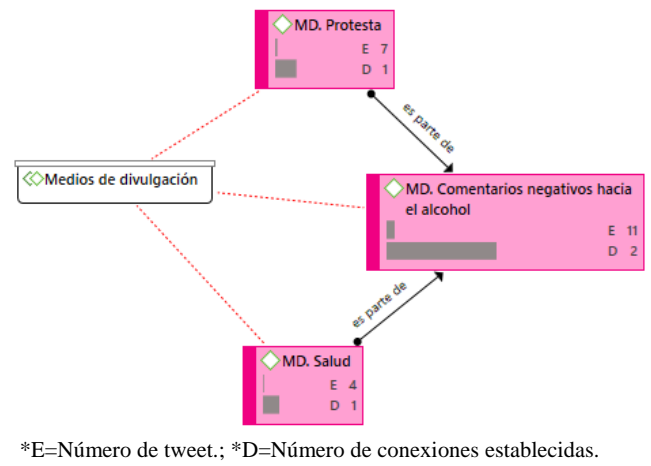

Del mismo modo, los medios divulgativos, así como la policía o los grupos de protesta, manifiestan comentario en contra del consumo de alcohol y alerta de las consecuencias negativas y el riesgo que supone para la salud el consumo excesivo de alcohol. Por su parte, los medios divulgativos, informan tanto de los efectos negativos que puede producir para su salud $(n=4)$, así como en forma de protesta por los daños que ocasiona el botellón en la vía pública $(n=7)$.

Figura 3. Tipos de comentarios por parte de los grupos de protesta en Twitter

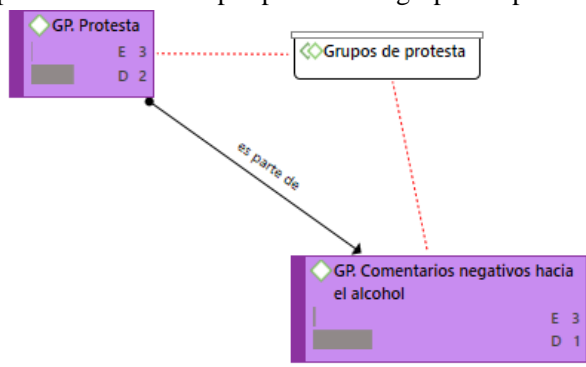

*E=Número de tweet.; *D=Número de conexiones establecidas. 
En cuanto a los grupos de protesta, forman perfiles de twitter con el objetivo de mostrar a la sociedad y concretamente a los jóvenes de las consecuencias que tiene el consumo no solo para la salud, sino para que sean consciente de los efectos nocivos que tiene para la sociedad en general $(n=3$ ) (Figura 3). Por último, los cuerpos y fuerzas de seguridad del estado, también muestran sus opiniones en contra de la ingesta de alcohol, ayudándose de las redes sociales para tal fin (14) (Figura 4).

Figura 4. Tipos de comentarios por parte de los policías en Twitter

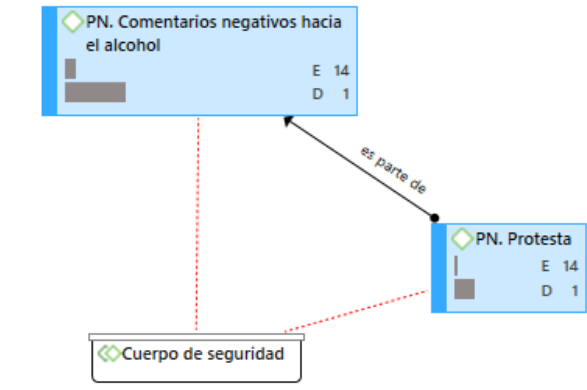

*E=Número de tweet.; *D=Número de conexiones establecidas.

Figura 5a. Tweets sobre medidas de prevención sobre el consumo

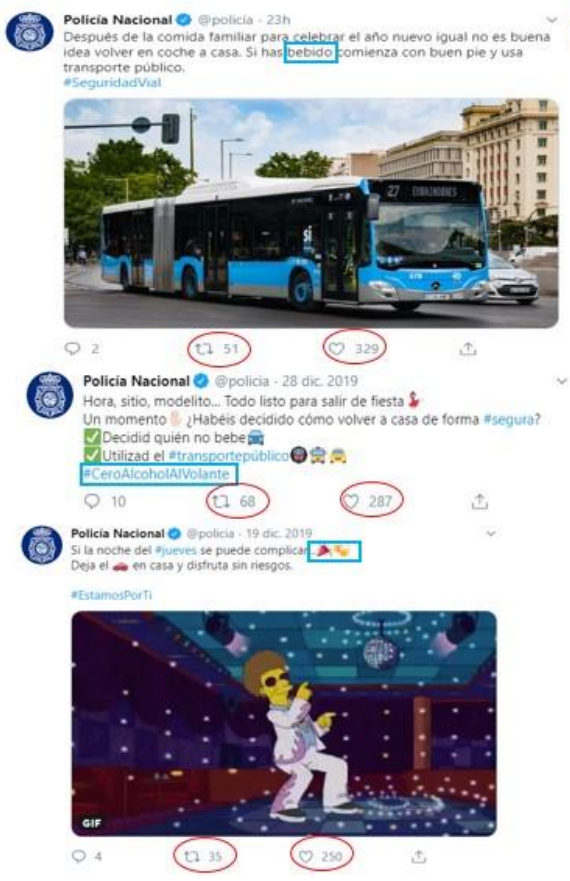

(2)

Policia Nacional 0 epolicia - 25 dic 2019

Hoy toca comida con la tamilias y unas copas "if para celebrar las

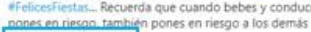
equiridedvial nanes en riscan tambien pones en riesgo a los demis
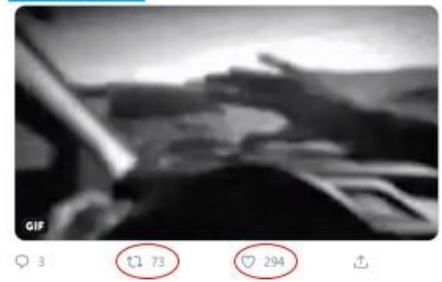

(2)

Policia Nacional 0 epolicia - 19 dic. 2019 Ya estamos celebrando que se acerca el 2020 ;

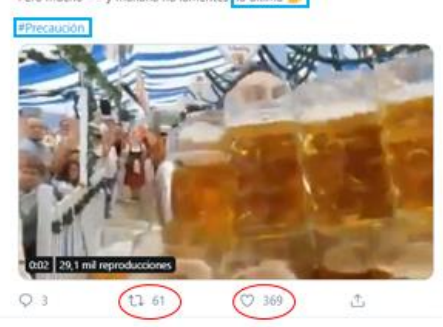


PÉREZ-FUENTES et al. Análisis de las redes sociales en el consumo de alcohol

Figura $5 b$. Tweets sobre medidas de prevención sobre el consumo

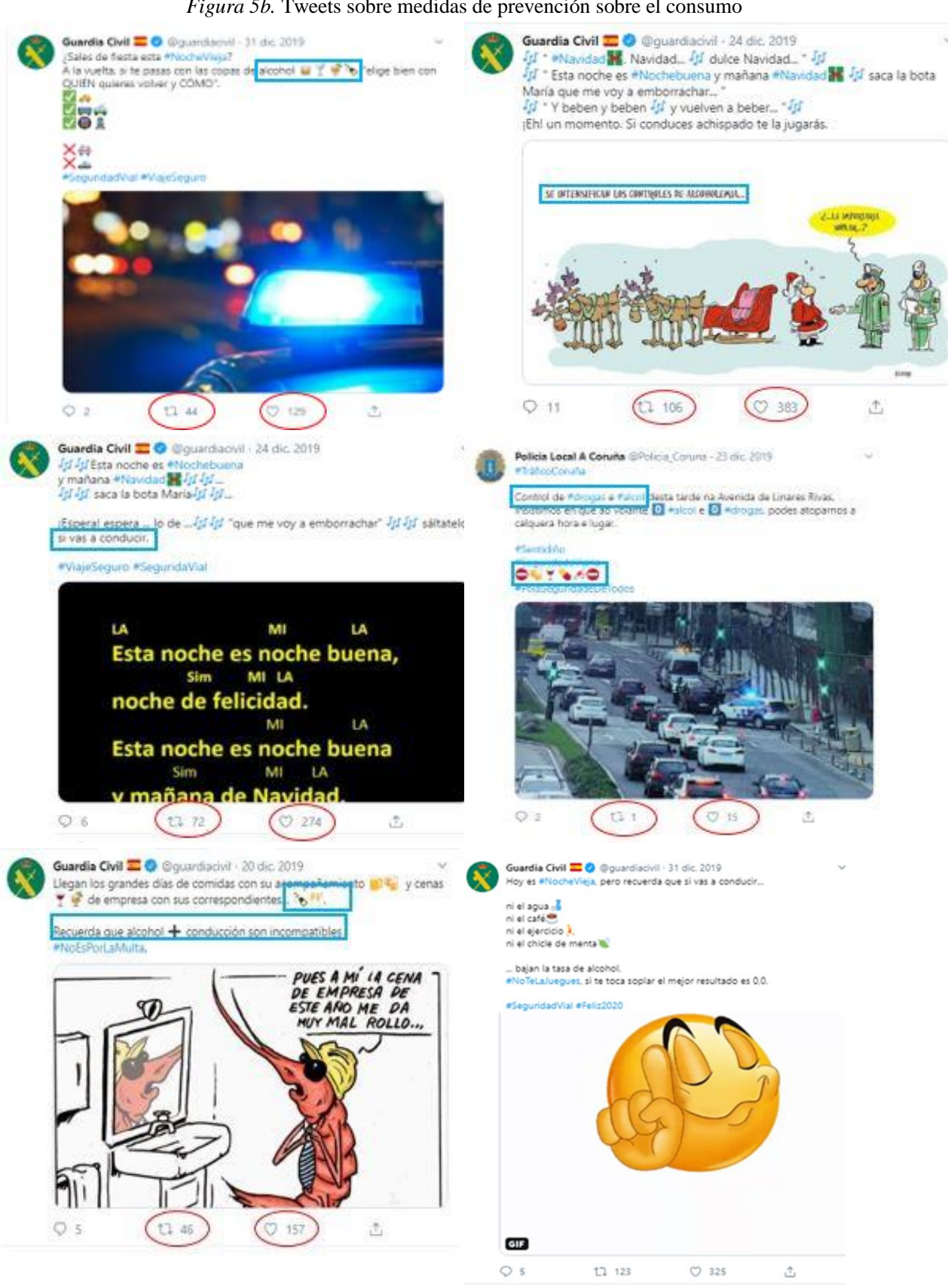


Así bien, se han encontrado diferentes tweets en los cuales la Policía Nacional, así como la Guardia civil utiliza la red social como herramienta de prevención del consumo de alcohol, compartiendo ideas para no coger el coche si los usuarios ingieren alcohol (Figura 5a y 5b). Como se observa, cada uno de los tweets tienen una gran repercusión, conteniendo un gran número de retweet y me gustas a favor de este tipo de comentarios contra la conducción bajo los efectos del alcohol.

En esta misma línea, se han encontrado diferentes tweets de diferentes medios de divulgación, los cuáles usan Twitter como herramienta de prevención del consumo de alcohol, compartiendo noticias sobre las consecuencias del consumo, así como las prohibiciones y sanciones que puede generar la ingesta del mismo.

\section{Figura 6. Sanciones}
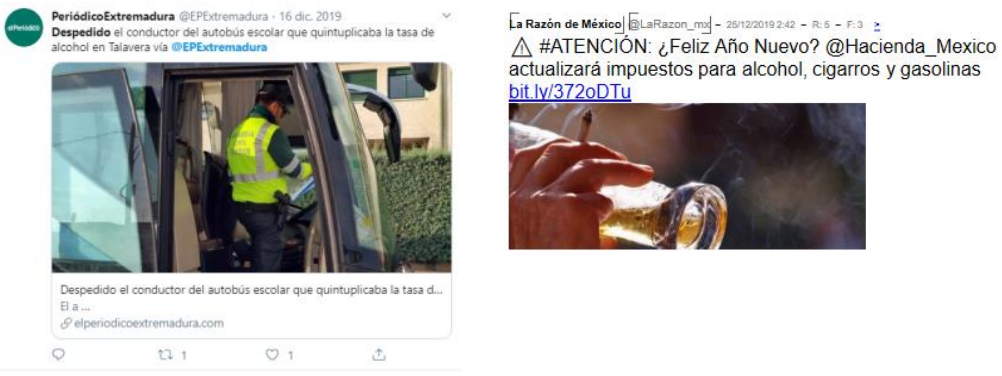

Figura 7. Protestas

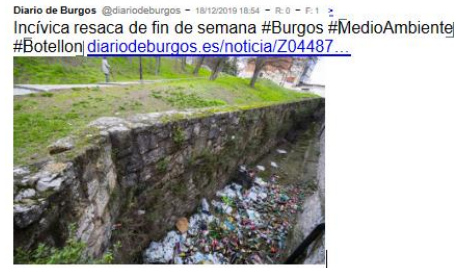

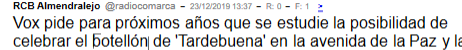
celebrar el Dotellón de 'Tardebuena' en la avenida de la
plaza de la Libertad, según informa en un comunicado.

Figura 8. Influencia del alcohol en la salud
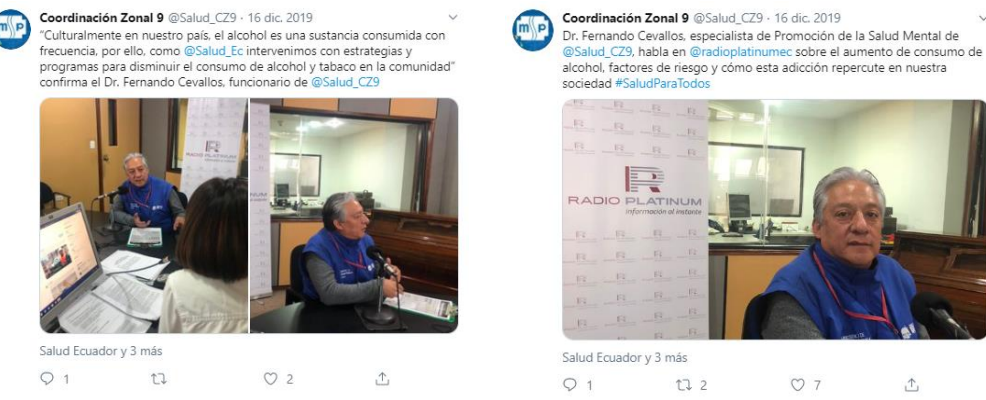
Igualmente, se corrobora la importancia de las palabas clave en los trabajos científicos, ya que facilita visualizar los términos más populares en las redes sociales. Por ello, en la figura 9 se puede observar como el tamaño de los diferentes conceptos que aparecen en la nube de palabras representan la relevancia que tiene cada una de las palabras clave relacionadas con el número de tweets que las contiene. Las palabras que se pueden observar en la nube de palabras están relacionadas con el consumo de alcohol en los diferentes idiomas utilizados para el presente trabajo. Entre las palabras que más menciones tiene podemos encontrar Alcohol (171), Botellón (128), Borracho (47), fiesta (5), y en menor medida palabras como tratamiento, suciedad, legalización entre otras con una sola entrada.

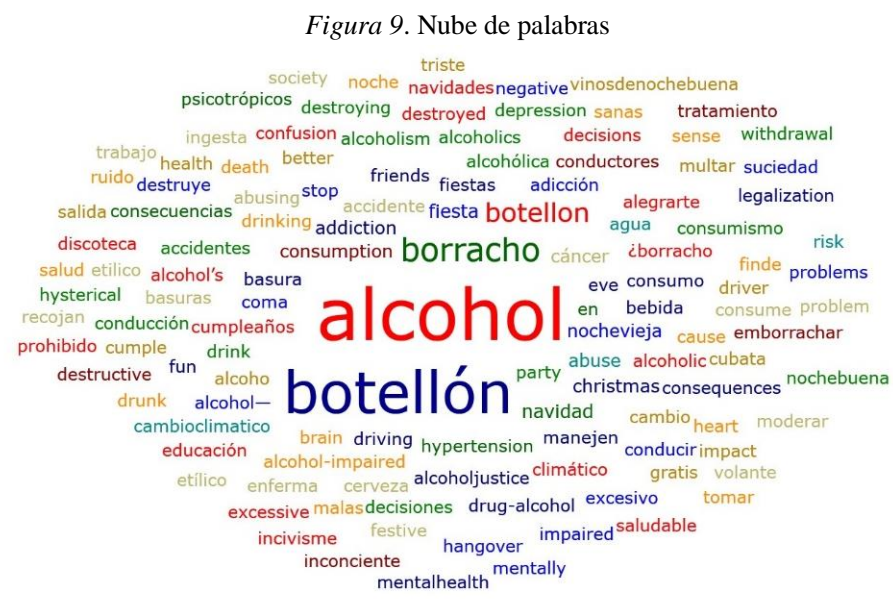

En cuanto a la tabla 2, encontramos el número de citas halladas por documento en función de la clasificación por grupos realizadas y el descriptor utilizado para la búsqueda. En dicha tabla, se puede observar como el documento llamado botellón es donde se encuentra el mayor número de citas (191), esto se ve influido por la época en la que se encuentra enfocado el presente trabajo, así como que el botellón es una práctica muy frecuente entre los jóvenes.

Tabla 2. Número de citas en función del descriptor

\begin{tabular}{|c|c|c|c|c|c|c|}
\hline & $\begin{array}{c}\text { Importación } \\
\text { Twitter: \#botellón }\end{array}$ & Bebida & $\begin{array}{c}\text { Importación } \\
\text { Twitter: botellón }\end{array}$ & $\begin{array}{c}\text { Importación } \\
\text { Twitter: borracho }\end{array}$ & $\begin{array}{c}\text { Importación } \\
\text { Twitter: alcohol }\end{array}$ & Total \\
\hline Medios divulgación & 1 & 7 & 6 & 0 & 0 & 14 \\
\hline Cuerpos seguridad & 2 & 2 & 0 & 0 & 0 & 4 \\
\hline Grupos de protesta & 3 & 0 & 2 & 0 & 0 & 5 \\
\hline Usuarios & 8 & 207 & 183 & 56 & 58 & 512 \\
\hline Totales & 14 & 216 & 191 & 56 & 58 & 535 \\
\hline Fecha publicación & $11 / 12 / 19-19 / 12 / 19$ & $15 / 12 / 19-16 / 12 / 19$ & $23 / 12 / 19-24 / 12 / 19$ & $24 / 12 / 19$ & $25 / 12 / 19$ & \\
\hline
\end{tabular}


Por otro lado, se han seleccionado una muestra representativa de los tweets analizados, para corroborar si la repercusión de los tweets sobre el consumo de alcohol depende del número de retweets, me gustas y el número de seguidores de los usuarios. Así bien, se observa como aquellos que tienen un mayor número de seguidores, sus entradas tienen un mayor número de retweets y me gustas, sobre todo en el caso de aquellos usuarios que son gamers, youtubers, influencers, personajes públicos, así como, instituciones, medios de divulgación o cuentas pertenecientes a grupos de seguridad del estado (Figura 10).

Figura 10. Repercusión de los tweets sobre el consumo de alcohol

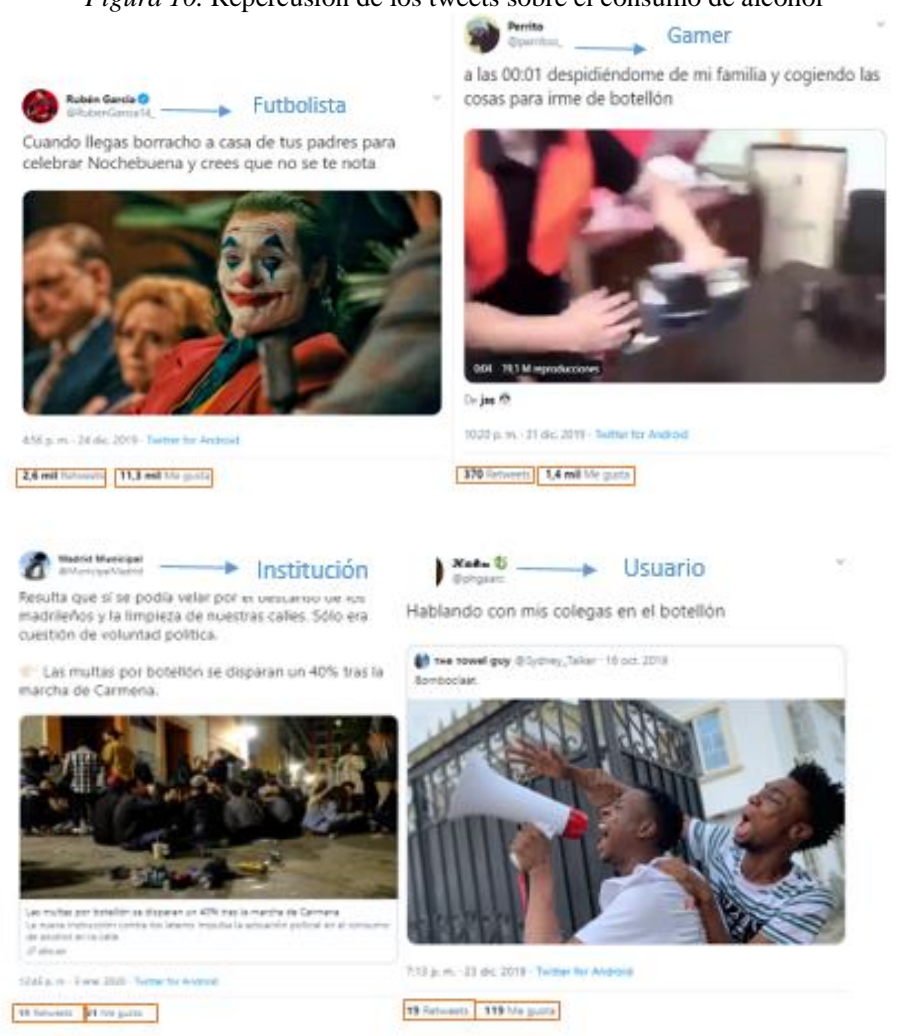




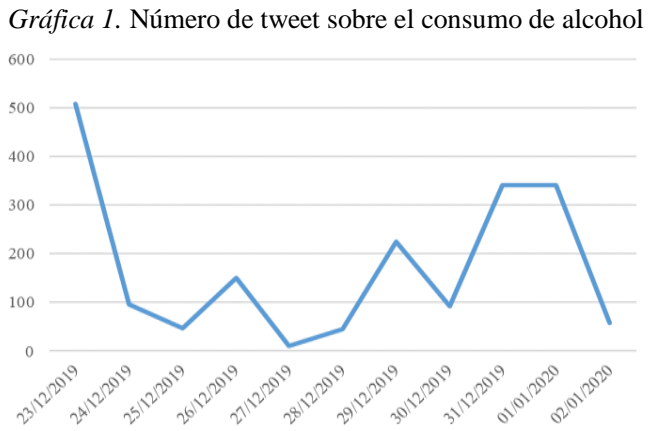

En la gráfica 1, se compararon dos semanas: del 23 de diciembre de 2019 al 1 de enero de 2020, coincidiendo con las festividades navideñas donde se corrobora como los días previos a días festivos, así como el día festivo, aumenta el número de comentarios sobre la ingesta de alcohol, es por ello, por lo que los días 23 de diciembre (508), 31 de diciembre (342) y 1 de enero (342), observamos un elevado número de tweet, disminuyendo progresivamente en los días continuos, el 24 de diciembre (96), 25 de diciembre (47), el 26 de diciembre (151), 29 de diciembre (224), y 30 de diciembre (92).

\section{DICUSIÓN Y CONCLUSIONES}

Las redes sociales se han convertido en la actualidad en una herramienta de comunicación en línea a tiempo real, donde los usuarios pueden compartir opiniones, ideas, e información (Moreno et al., 2016). Así, gracias aplicaciones como Facebook o Twitter, se puede observar el consumo de alcohol en jóvenes (Beullens y Schepers, 2013; Moreno et al., 2012).

Tras analizar los resultados, se aprecia como los jóvenes están a favor del consumo de alcohol debido a los comentarios positivos que muestran en las redes sociales (Cavazos-Rehg et al., 2015; West et al., 2012), mientras que el número de comentarios en contra del consumo de alcohol y de prevención es menor (Litt et al., 2018), confirmándose así la primera hipótesis del presente trabajo.

Por otro lado, con la ayuda de las redes no solo son usuarios los que pueden mostrar sus opiniones en este tipo de plataformas, sino que, desde las instituciones, el gobierno, los cuerpos de seguridad del estado, los medios de comunicación, así como los políticos pueden expresarse a través de estas redes, realizando campañas de concienciación y prevención del consumo de alcohol donde pueden llegar a todo el colectivo (Kersbergen y Field, 2017; Stautz et al., 2016). Se hace imprescindible, 
compartir las consecuencias que produce para la salud la ingesta incontrolada de alcohol tanto a corto como a largo plazo.

Asimismo, en relación a la época donde más se muestra el consumo de alcohol, se aprecia una relación entre el aumento del consumo de alcohol y los días festivos. Es decir, los días previos a días festivos se aprecia un aumento de publicaciones en redes sociales en relación a la ingesta de alcohol (Bellis et al., 2015).

Los hallazgos obtenidos tras el análisis de los resultados pueden resultar de interés para conocer la presencia que tiene el consumo de alcohol en las redes sociales. No obstante, en el desarrollo del presente trabajo se han encontrado algunas limitaciones. Los idiomas utilizados para la búsqueda fueron el español y el inglés, es por ello, por lo que los resultados no se pueden generalizar ya que no se tuvieron en cuenta los comentarios de usuarios realizados en otro idioma. Otra limitación encontrada es la porción de datos analizada, respecto a la gran cantidad de datos de Twitter y Facebook. Por otro lado, aunque se ha intentado reducir los sesgos de las publicaciones sobre el consumo de alcohol en redes sociales, eliminando los comentarios duplicados y mediante una lectura crítica se eliminaron aquellos que no aportaban información relevante al estudio, se deben tener en cuenta los sesgos de las entradas incluidas en el presente trabajo.

De los resultados expuestos anteriormente, se pueden derivar futuras líneas de investigación. Pudiendo ser de interés aumentar el número de entradas en Facebook y Twitter analizadas, de esta manera recogeremos resultados más amplios de dicha investigación. Así mismo, se podría incluir una variable más al estudio, como el consumo de tabaco o cannabis.

Las redes sociales se han convertido en la nueva forma de comunicarse entre los jóvenes, lo que facilita el intercambio de opiniones, ideas e información a nivel mundial. Así, han posibilitado observar como las redes sociales influyen en el consumo y la prevención del alcohol, por tanto, las redes pueden ser utilizadas como herramientas para realizar campañas y programas de prevención del consumo de alcohol en los jóvenes, reduciendo la ingesta del mismo.

El presente trabajo refleja la repercusión que tienen las redes sociales en el desarrollo de los jóvenes en cuanto al consumo de alcohol, mostrando como en la mayoría de los casos se muestran a favor del alcohol, como un medio esencial para disfrutar y pasarlo bien. Sin embargo, se pone de manifiesto la escasa investigación existente sobre el consumo de alcohol en redes sociales.

\section{Agradecimientos}

El presente trabajo se ha desarrollado gracias al Proyecto Violencia entre iguales y consumo de alcohol y tabaco en Educación Secundaria: programa basado en realidad aumentada para la detección e intervención (Referencia: EDU2017-88139-R), 
financiado por el Programa Estatal de Investigación, Desarrollo e Innovación Orientada a los Retos de la Sociedad, en el marco del Plan Estatal de Investigación Científica y Técnica y de Innovación, y la cofinanciación con Fondos Estructurales de la Unión Europea.

\section{REFERENCIAS}

Alonso-Fernández, N., Jiménez-Trujillo, I., Hernández-Barrera, V., Palacios-Ceña, D., y Carrasco-Garrido, P. (2019). Alcohol Consumption Among Spanish Female Adolescents: Related Factors and National Trends 2006-2014. International Journal of Environmental Research and Public Health, 16(21), 4294. doi:10.3390/ijerph16214294

Bellis, M.A., Hughes, K., Jones, L., Morleo, M., Nicholls, J., McCoy, E., ... Sumnall, H. (2015). Holidays, celebrations, and commiserations: measuring drinking during feasting and fasting to improve national and individual estimates of alcohol consumption. BMC Medicine, 13, 113. doi:10.1186/s12916-015-0337-0

Beullens, K., y Schepers, A. (2013). Display of Alcohol Use on Facebook: A Content Analysis. Cyberpsychology, Behavior, and Social Networking, 16(7), 497-503. doi:10.1089/cyber.2013.0044

Bowden, J.A., Delfabbro, P., Room, R., Miller, C.L., y Wilson, C. (2017). Prevalence, perceptions and predictors of alcohol consumption and abstinence among South Australian school students: a cross-sectional analysis. BMC Public Health, 17(1), 549. doi:10.1186/s12889-017-4475-5

Boyle, S.C., LaBrie, J.W., Froidevaux, N.M., y Witkovic, Y.D. (2016). Different digital paths to the keg? How exposure to peers' alcohol-related social media content influences drinking among male and female first-year college students. Addictive Behaviors, 57, 21-29. doi:10.1016/j.addbeh.2016.01.011

Cavazos-Rehg, P.A., Krauss, M.J., Sowles, S.J., y Bierut, L.J. (2015). "Hey everyone, I'm drunk.” An evaluation of drinking-related Twitter chatter. Journal of Studies on Alcohol and Drugs, 76(4), 635-643. doi:10.15288/jsad.2015.76.635

Cutrín, O., Maneiro, L., Sobral, J., y Gómez-Fraguela, J.A. (2019). Longitudinal effects of parenting mediated by deviant peers on violent and non-violent antisocial behaviour and substance use in adolescence. The European Journal of Psychology Applied to Legal Context, 11, 23-32. doi:10.5093/ejpalc2018a12

De la Fuente, I.I., Rodríguez, A., y Escalante, N. (2019). "Apoyo social percibido e implicación escolar: Correlaciones y variabilidad". European Journal of Child Development, Education and Psychopathology, 7(1), 23-35. doi:10.30552/ejpad.v7i1.86

Dumont, S., Marques-Vidal, P., Favrod-Coune, T., Theler, J. M., Gaspoz, J.M., Broers, B., y Guessous, I. (2017). Alcohol policy changes and 22-year trends in individual alcohol consumption in a Swiss adult population: a 1993-2014 cross-sectional population-based study. BMJ Open, 7(3), e014828. doi:10.1136/bmjopen-2016-014828

ESTUDES (2019). "Encuesta sobre uso de drogas en enseñanzas secundarias en España 20182019." Ministerio de Sanidad, Consumo y Bienestar Social Secretaría de Estado de Servicios Sociales. Delegación del Gobierno para el Plan Nacional sobre Drogas.

Fernández-Lasarte, O., Ramos-Díaze, E., y Axpe, I. (2019). "Rendimiento académico, apoyo social percibido e inteligencia emocional en la universidad". European Journal of Investigation in Health, Psychology and Education, 9(1), 39-49. doi:10.30552/ejihpe.v9i1.315 
Gázquez, J., Pérez-Fuentes, M., Carrión, J., Luque, A., y Molero, M. (2015). Interpersonal Value Profiles and Analysis to Adolescent Behavior and Social Attitudes. Revista de Psicodidáctica, 20(2), 321-337. doi:10.1387/RevPsicodidact.12978

Hall, W.D., Patton, G., Stockings, E., Weier, M., Lynskey, M., Morley, K., y Degenhardt, L. (2016). Why young people's substance use matters for global health. The Lancet Psychiatry, 3(3), 265-279. doi:10.1016/S2215-0366(16)00013-4

Hendriks, H., Van den Putte, B., Gebhardt, W.A., y Moreno, M.A. (2018). Social Drinking on Social Media: Content Analysis of the Social Aspects of Alcohol-Related Posts on Facebook and Instagram. Journal of Medical Internet Research, 20(6), e226. doi:10.2196/jmir.9355

Huertas, E., López-Moreno, J.A., Fernández, V., Echeverry-Alzate, V., y Bühler, K.M. (2019). Associations between experimental substance use, FAAH-gene variations, impulsivity and sensation seeking. Psicothema, 31(3), 239-245. doi:10.7334/psicothema2019.27

Jorge, K., Ferreira, R., Ferreira, E., Vale, M., Kawachi, I., y Zarzar, P. M. (2017). Binge drinking and associated factors among adolescents in a city in southeastern Brazil: a longitudinal study. Cadernos de Saúde Pública, 33(2), e00183115. doi:10.1590/0102-311x00183115

Kersbergen, I., y Field, M. (2017). Visual attention to alcohol cues and responsible drinking statements within alcohol advertisements and public health campaigns: Relationships with drinking intentions and alcohol consumption in the laboratory. Psychology of Addictive Behaviors: Journal of the Society of Psychologists in Addictive Behaviors, 31(4), 435-446. doi:10.1037/adb0000284

Ley 5/2018, de 3 de mayo, de prevención del consumo de bebidas alcohólicas en la infancia y la adolescencia.

Litt, D.M., Lewis, M.A., Spiro, E.S., Aulck, L., Waldron, K.A., Head-Corliss, M.K., y Swanson, A. (2018). \#drunktwitter: Examining the relations between alcohol-related Twitter content and alcohol willingness and use among underage young adults. Drug and Alcohol Dependence, 193, 75-82. doi:10.1016/j.drugalcdep.2018.08.021

López, S., y Rodríguez-Arias, J.L. (2010). "Factores de riesgo y de protección en el consumo de drogas en adolescentes y diferencias según edad y sexo". Psicothema, 22(4), 568-573.

Lu, W., Xu, J., Taylor, A.W., Bewick, B.M., Fu, Z., Wu, N., ... Yin, P. (2019). Analysis of the alcohol drinking behavior and influencing factors among emerging adults and young adults: a cross-sectional study in Wuhan, China. BMC Public Health, 19(1), 458. doi:10.1186/s12889-019-6831-0

Maganto, C., Peris, M., y Sánchez, R. (2019). "El bienestar psicológico en la adolescencia: variables psicológicas asociadas y predictoras". European Journal of Education and Psychology, 12(2), 139-151.

Martínez-Loredo, V., Fernández-Hermida, J.R., de La Torre-Luque, A., y Fernández-Artamendi, S. (2018). Polydrug use trajectories and differences in impulsivity among adolescents. International Journal of Clinical and Health Psychology, 18(3), 235-244. doi:10.1016/j.ijchp.2018.07.003

Meier, P.S., Holmes, J., Angus, C., Ally, A.K., Meng, Y., y Brennan, A. (2016). Estimated Effects of Different Alcohol Taxation and Price Policies on Health Inequalities: A Mathematical Modelling Study. PLoS Medicine, 13(2), e1001963. doi:10.1371/journal.pmed.1001963

Molina, P.E., y Nelson, S. (2018). Binge Drinking's Effects on the Body. Alcohol Research: Current Reviews, 39(1), 99-109.

Moore, S.C., Alam, F., Heikkinen, M., Hood, H., Huang, C., Moore, L., Murphy, S., Playle, R., Shepherd, J., Shovelton, C., Sivarajasingam, V., y Williams, A. (2017). The effectiveness of an intervention to reduce alcohol-related violence in premises licensed 
for the sale and on-site consumption of alcohol: a randomized controlled trial. Addiction, 112, 1898-1906. doi:10.1111/add.13878

Moreno, M.A., Christakis, D.A., Egan, K.G., Brockman, L.N., y Becker, T. (2012). Associations between displayed alcohol references on Facebook and problem drinking among college students. Archives of Pediatrics \& Adolescent Medicine, 166(2), 157-163. doi:10.1001/archpediatrics.2011.180

Moreno, M.A., Cox, E.D., Young, H.N., y Haaland, W. (2015). Underage college students' alcohol displays on Facebook and real-time alcohol behaviors. The Journal of Adolescent Health: Official Publication of the Society for Adolescent Medicine, 56(6), 646-651. doi:10.1016/j.jadohealth.2015.02.020

Moreno, M.A., D'Angelo, J., y Whitehill, J. (2016). Social media and alcohol: summary of research, intervention ideas and future study directions. Media and Communication, 4(3), 50-59. doi:10.17645/mac.v4i3.529.

Murphy, E., O'Sullivan, I., O'Donovan, D., Hope, A., y Davoren, M.P. (2016). The association between parental attitudes and alcohol consumption and adolescent alcohol consumption in Southern Ireland: a cross-sectional study. BMC Public Health, 16(1), 821. doi:10.1186/s12889-016-3504-0

Nelson, J.P., y McNall, A.D. (2016). Alcohol prices, taxes, and alcohol-related harms: a critical review of natural experiments in alcohol policy for nine countries. Health Policy, 120(3), 264-272. doi:10.1016/j.healthpol.2016.01.018

OMS (2018). "Factores que influyen en el consumo de alcohol y los daños relacionados con el alcohol".

Parada, M., Corral, M., Caamaño-Isorna, F., Mota, N., Crego, A., Rodríguez, S., y Cadaveira, F. (2011). "Definición del concepto de consumo intensivo de alcohol adolescente (binge drinking)". Adicciones, 23(1), 53-63. doi:10.20882/adicciones.167

Pérez-Fuentes, M.C., Gázquez, J.J., Molero, M.M., Cardila, F., Martos, A., Barragán, A.B., Garzón, A., Carrión, J.J., y Mercader, I. (2015). "Impulsividad y consumo de alcohol y tabaco en adolescentes". European Journal of Investigation in Health, Psychology and Education, 5(3), 371-382. doi:10.1989/ejihpe.v5i3.139

Pérez-Fuentes, M.C., Molero, M.M., y Gázquez, J.J. (2018). Expectations and Sensation-Seeking as predictors of Binge Drinking in adolescents. Annals of Psychology, 35(1), 124-130. doi: 10.6018/analesps.35.1.308511

Piano, M.R. (2017). Alcohol's Effects on the Cardiovascular System. Alcohol Research: Current Reviews, 38(2), 219-241.

Rehm, J., Gmel, G.E., Gmel, G., Hasan, O., Imtiaz, S., Popova, S., ... Shuper, P.A. (2017). The relationship between different dimensions of alcohol use and the burden of disease-an update. Addiction, 112(6), 968-1001. doi:10.1111/add.13757

Sebena, R., Orosova, O., Mikolajczyk, R.T., y Van Dijk, J.P. (2011). Selected sociodemographic factors and related differences in patterns of alcohol use among university students in Slovakia. BMC Public Health, 11(849).

Stautz, K., Brown, K.G., King, S.E., Shemilt, I., y Marteau, T.M. (2016). Immediate effects of alcohol marketing communications and media portrayals on consumption and cognition: a systematic review and meta-analysis of experimental studies. BMC Public Health, 16, 465. doi:10.1186/s12889-016-3116-8

West, J., Hall, P., Hanson, C., Prier, K., Giraud-Carrier, C., Neeley, E., y Barnes, M. (2012). Temporal variability of problem drinking on Twitter. Open Journal of Preventive Medicine, 2(1), 43-48.

Zadarko-Domaradzka, M., Barabasz, Z., Sobolewski, M., Nizioł-Babiarz, E., Penar-Zadarko, B., Szybisty, A., y Zadarko, E. (2018). Alcohol Consumption and Risky Drinking Patterns 
among College Students from Selected Countries of the Carpathian Euroregion. Biomed Research International, 2018, 6084541. doi:10.1155/2018/6084541

Recibido: 4 de agosto de 2020 Recepción Modificaciones: 14 de septiembre de 2020 Aceptado: 16 de septiembre de 2020 